\title{
A Full-duplex Access Network based on CWDM-routed PONs
}

\author{
Y. Shachaf, P. Kourtessis, J. M. Senior \\ Optical Networks Group, Science and Technology Research Institute (STRI), University of Hertfordshire, College Lane Campus, Hatfield, \\ AL10 9AB, UK. +44(0) 1707285082.y.shachaf@herts.ac.uk
}

\begin{abstract}
The application of polarisation-division-multiplexing in presented, demonstrating full-duplex transmission in an access network architecture employing RSOA-based ONUs. Modelling of a single coarse-AWG OLT to transmit orthogonally-multiplexed burst-data and continuous-waves demonstrated crosstalk-free transmission up to $20 \mathrm{~km}$.

(C)2008 Optical Society of America

OCIS codes (060.0060) Fiber optics and optical communications; (060.4250) Networks; (260.5430) Polarization; (060.4230) Multiplexing; (230.5440) Polarization-sensitive devices
\end{abstract}

\section{Introduction}

The escalating demand for bandwidth, driven primarily by advances in broadband services, e.g. high definition TV, video-on-demand, user-generated content requires upgrading the access network infrastructure. Passive optical networks (PONs) have gained considerable support in providing the final wired solution, capable of achieving symmetrical service provisioning bandwidths ranging from $30 \mathrm{Mbit} / \mathrm{s}$ to more than $50 \mathrm{Mbit} / \mathrm{s}$ [1].

A scalable access network architecture has been developed featuring interoperability among time division multiplexing (TDM) and wavelength division multiplexing (WDM) PONs by means of a coarse passband array waveguide grating (AWG)-based optical line terminal (OLT) in the presence of polarisation-dependent wavelength (PDW) shifting and phase errors [2]. This letter proposes the application of polarisation division multiplexing (PDM) [3] and colourless optical network units (ONUs), by employing reflective semiconductor optical amplifiers (RSOAs), to demonstrate full-duplex transmission over the formerly developed access network architecture [2] and subsequently increase bandwidth utilisation in both upstream and downstream.

This is achieved by splitting the original continues waves (CWs) into orthogonal polarisations, one of which is modulated for downstream data transmission and the other fed to an RSOA for upstream transmission. Consequently the proposed scheme overcomes time-interleaving in the OLT of the transmission time-slots between CWs and burst-data allowing for their independent transmission over the two polarisations to avoid multiplexing idle time. Assuming the use of symmetrical broadband services such as video conferencing and online gaming [4], the proposed scheme could potentially double the bandwidth utilisation for each subscriber, resulting in increased network upstream and downstream throughput.

\section{Network architecture}

The network architecture, shown in Fig. 1, exhibits a $4 \times 4$ coarse AWG in the OLT to route multiple TDM and WDM-PONs by means of $7 \mathrm{~nm}$ Gaussian passband windows [5]. To serve all ONUs of TDM-PON1, tunable laser (TL1) will optimally utilise the wavelength $\lambda_{1}{ }^{9}$, placed at the centre of the AWG coarse channel $\lambda_{1}$. To serve all ONUs of WDM-PON4, TL1 will utilise $16,0.4 \mathrm{~nm}$-spaced wavelengths $\lambda_{2}{ }^{1-16}$, centred $\pm 3.2 \mathrm{~nm}$ around coarse channel $\lambda_{2}$ [2] featuring a smooth migration from single to multi-wavelength optical access to address increasing bandwidth requirements. The use of a single TL in the OLT allows for cross operational management of network load by dynamically assigning downstream wavelengths to each PON in tandem with CWs for upstream transmission in the RSOAs [2]. Consequently, all ONUs served by TL1 downstream, will be

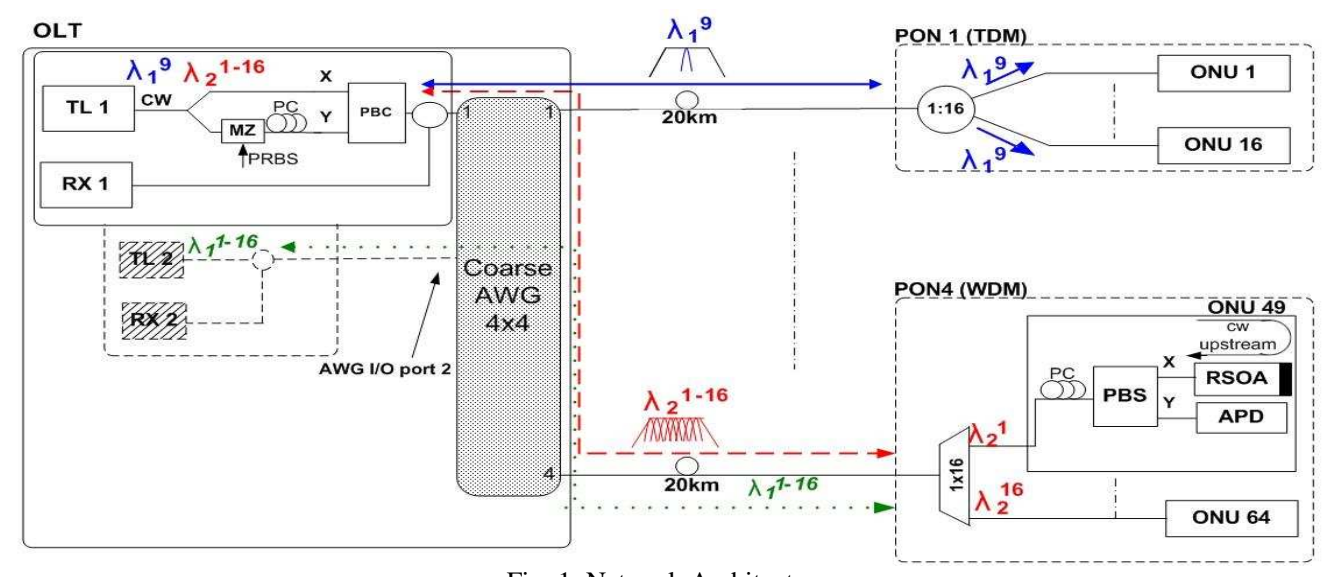

Fig. 1. Network Architecture 


\section{JThA101.pdf}

terminated upstream to a single receiver, RX1, possible due to the AWG's reciprocal nature. In addition, the network exhibits increased scalability since extra TLs can be directly applied at unused AWG ports in the OLT, e.g. TL2 at I/O port 2, to increase bandwidth provision at high traffic load [2].

As shown in Fig. 1, the CW of TL1 is initially split in an optical coupler. The lower coupler arm is applied at a Mach-Zehnder modulator (MZM) for downstream data modulation, consequently linearly-vertical-polarised (Y polarisation) by a polarisation controller. The upper arm, displaying the linearly-horizontally-polarised CW (X polarisation), is connected directly to a polarisation beam combiner (PBC) to be multiplexed with the orthogonal Y polarisation. Subsequently, the two orthogonally multiplexed signals are applied at first to a circulator to allow bidirectional transmission and depending on the operating wavelength, routed via the appropriate AWG coarse channel to the corresponding PON [2]. Once inside each TDM or WDM-PON ONU, the two orthogonal signals are applied to a polarisation controller to be aligned with the succeeding polarisation beam splitter (PBS) $\mathrm{x}-\mathrm{y}$ coordinates. After demultiplexing the $\mathrm{CW}$ polarisation is injected into an RSOA for upstream transmission, while the received data is terminated at an avalanche photodetector (APD), incapable of employing the RSOA for both simultaneous detection and modulation [2]. In upstream, each reflected modulated wave is routed through exactly the same path followed in downstream, and subsequently terminated in turn to a single receiver RX1 in the OLT.

\section{Network modelling and results}

The Virtual Photonics Inc. (VPI) simulation platform was used to investigate the effect of the coarse AWG Gaussian response in the power distribution, passband shifting and also the orthogonallity of the multiplexed signals. This was achieved by modelling WDM-PON4 since it has been demonstrated to employ the most and least severely degraded ONUs by means of PDW and polarisation-dependent loss (PDL) [2], corresponding to the longest and centre wavelengths at $\lambda_{2}{ }^{1}=1553.33 \mathrm{~nm}$ and $\lambda_{2}{ }^{9}=1550.12 \mathrm{~nm}$ of the AWG passband respectively.

For that purpose, TL1 at $+1 \mathrm{dBm}$ was applied at a commercially-available 1:97 coupler to accommodate the network wavelength-specific losses, in the range of $13 \mathrm{~dB}$ to $19.5 \mathrm{~dB}$, and to allow sufficient $\mathrm{CW}$ downstream power while avoiding to saturate the RSOA's $-29 \mathrm{dBm}$ input figure. At the upper coupler arm, each CW at approximately $0 \mathrm{dBm}$ was modulated by a $1.25 \mathrm{Gbit} / \mathrm{s}, 2^{7}-1$ long PRBS to comply with the limited RSOA electrical frequency response at $900 \mathrm{MHz}$, in contrast to the lower coupler arm CWs at $-13 \mathrm{dBm}$ due to the 1:97 splitting ratio. Following multiplexing at the PBC, the orthogonal signals of each wavelength were applied to a circulator and via the AWG downstream input/output (I/O) port 4 coupled to $20 \mathrm{~km}$ of standard single-mode fibre (SSMF). At the network distribution point, a 1x16 demultiplexer transmitted the optical signals to the designated ONUs of WDM-PON.

In each ONU, the reflected modulated wavelength at a power ranging from $-6 \mathrm{dBm}$ to $0 \mathrm{dBm}$ due to the RSOA transverse-electric (TE) gain of $29 \mathrm{~dB}$, to eradicate polarisation dependent gain (PDG), and its position in the AWG passband, was in turn modulated by another random sequence with the same length and data rate to demonstrate upstream transmission at excitation ratio of $12 \mathrm{~dB}$.

The AWG induced PDW shift has been measured to impose a PDL of up to $3 \mathrm{~dB}$ [2] that could potentially degrade the degree of orthogonallity of the multiplexed signals if they are not aligned with the device TE and transverse-magnetic (TM) modes [6]. In the proposed architecture the AWG is located in the OLT and as a result the two states of polarisations (SOPs) are already aligned with the device input transmission modes, preserving their degree of orthogonallity. In contrast, after transmission through the SSMF the multiplexed signals reaching the distribution point display random but mutually orthogonal SOPs due to the fibre birefringence [6]. The demultiplexing device itself in a standard WDM PON distribution point has shown not to impose any significant effect on the degree of orthogonallity as a result of its typical 0.5 dB PDL [6].

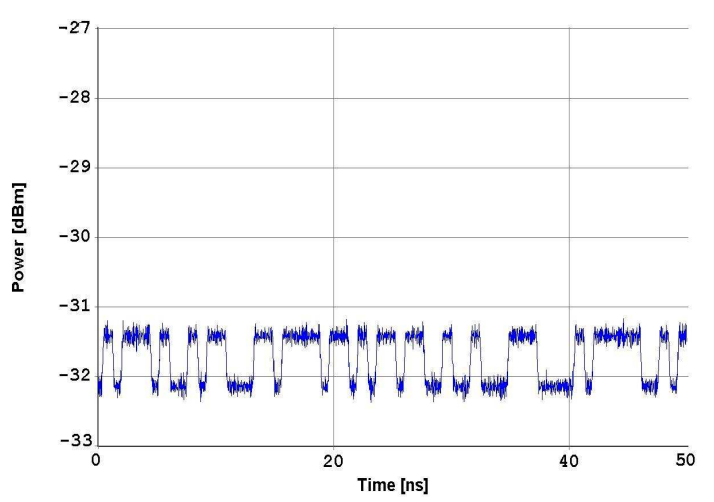

(a)

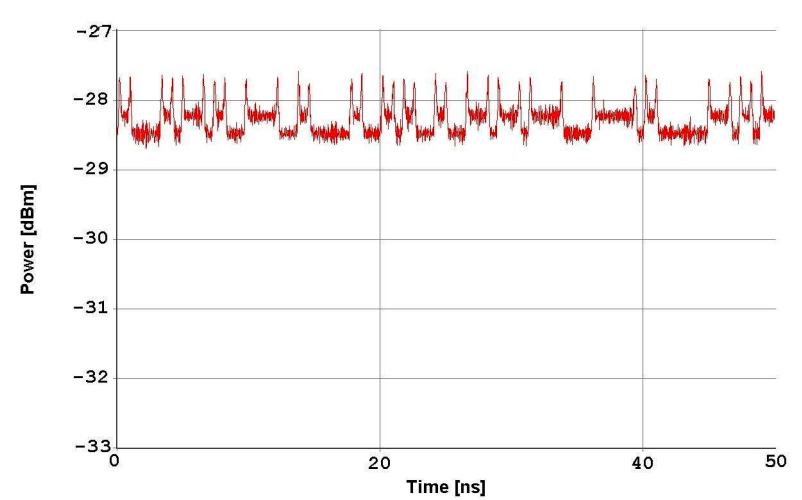

(b)

Fig. 2. Injected CW at RSOA optical input (a) $\lambda_{2}{ }^{1}$, (b) $\lambda_{2}{ }^{9}$ 


\section{JThA101.pdf}

In defining error-free detection downstream and subsequently optimum modulation in the RSOA, the crosstalk characteristic between the transmitted data and $\mathrm{CW}$ for the AWG worst-case and central wavelengths at $\lambda_{2}{ }^{1}=1553.33 \mathrm{~nm}$ and $\lambda_{2}{ }^{9}=1550.12 \mathrm{~nm}$ respectively was monitored with the objective to maintain low interference among the two forms of transmission to allow minimum leakage of burst data in the RSOA injected wave. As shown in Fig. 2 an insignificant crosstalk figure of no more than $1 \mathrm{~dB}$ in power leakage is observed compared to the measured excitation ratio of $12 \mathrm{~dB}$ of each reflected, modulated wavelength. In addition, a 3 $\mathrm{dB}$ power difference can be also observed between the received CW signals due to the AWG Gaussian response.

To evaluate the full-duplex network performance, bit-error-rate (BER) responses were drawn to account for the most and least severely degraded wavelengths servicing WDM-PON4. Confirmed by Fig. 3, $10^{-9}$ BERs were achieved by all upstream ONUs at the expense of a maximum $2.5 \mathrm{~dB}$ power penalty between $\lambda_{2}{ }^{1}$ and $\lambda_{2}{ }^{9}$ due to the induced PMD crosstalk and AWG response. In downstream, Fig. 3 displays no penalty among the wavelengths due to a high measured crosstalk isolation of $26 \mathrm{~dB}$. To make evident the capability of the network to allow full-duplex operation with insignificant limitation due to crosstalk, BER responses under half-duplex transmission were also drawn with either the downstream data or CW disabled for the duration of the upstream and downstream transmissions respectively. Confirmed by Fig. 4, similar BER figures were achieved by all ONUs at the expense of $1.2 \mathrm{~dB}$ power penalty between $\lambda_{2}{ }^{1}$ and $\lambda_{2}{ }^{9}$ due to the AWG response. Contrasting the half-duplex network performance to its full-duplex operation it is concluded that an approximate $1.3 \mathrm{~dB}$ increase in power penalty is induced to all transmitted wavelengths in the later, allowing for effective simultaneous transmission of data by means of overcoming sharing of the available transmission time-slots.

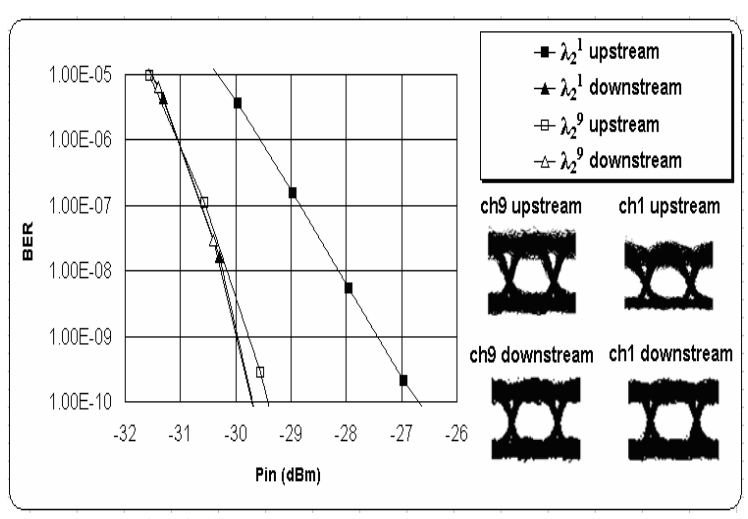

Fig. 3 BER for full-duplex bi-directional transmission

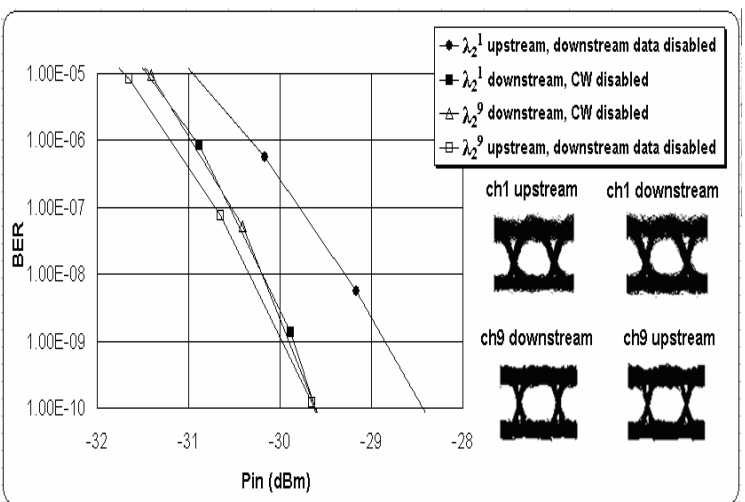

Fig. 4 BER for half-duplex upstream and downstream transmission

\section{Conclusion}

An orthogonally-multiplexed OLT has been demonstrated to achieve full-duplex operation in an access network architecture comprising RSOA-based ONUs and a single coarse-AWG routing device in the OLT by means of simultaneous data and CW transmission downstream to increase bandwidth utilisation. Transmission of the multiplexed $\mathrm{CW}$ and data signals through $7 \mathrm{~nm}$-wide passband windows of the AWG, has confirmed error-free transmission of all ONUs of a WDM-PON in the presence of a worst-case $3 \mathrm{~dB}$ PDL. Further investigations verified no more than $1 \mathrm{~dB}$ power leakage of the data-carrying polarisation onto the CWs, imposing no limitation in the network transmission efficiency when compared to a measured $12 \mathrm{~dB}$ excitation ratio of upstream modulated signals in the RSOA. In addition, contrasting between half and full-duplex operations has demonstrated a moderate $1.3 \mathrm{~dB}$ penalty superimposed at the later that still allows for $10^{-9}$ BER transmission at $1.25 \mathrm{Gbit} / \mathrm{s}$.

\section{References}

[1] P. Chanclou, S. Gosselin, J. F. Palacios, V. L. Álvarez, and E. Zouganeli, "Overview of the Optical Broadband Access Evolution: A Joint Article by Operators in the IST Network of Excellence e-Photon/ONe," IEEE Communications Magazine, vol. 44, pp. 29-35, 2006.

[2] Y. Shachaf, P. Kourtessis, and J. M. Senior, "An interoperable access network based on CWDM-routed PONs," presented at the 33rd European Conference and Exhibition on Optical Communication (ECOC), Berlin, Germany, 2007.

[3] I. Tsalamanis, E. Rochat, and S. D. Walker, "Experimental demonstration of cascaded AWG access network featuring bi-directional transmission and polarization multiplexing," OSA Optics Express, vol. 12, pp. 764-769, 2004.

[4] C.-H. Lee, S.-M. Lee, K.-M. Choi, J.-H. Moon, S.-G. Mun, K.-T. Jeong, J. H. Kim, and B. Kim, "WDM-PON experiences in Korea [Invited]," OSA Journal of Optical Networking, vol. 6, pp. 451-464, 2007.

[5] J. Jiang, C. L. Callender, C. Blanchetière, J. P. Noad, S. Chen, J. Ballato, and J. Dennis W. Smith, "Arrayed Waveguide Gratings Based on Perfluorocyclobutane Polymers for CWDM Applications," IEEE Photonics Technology Letters, vol. 18, pp. 370-372, 2006.

[6] I. Tsalamanis, E. Rochat, M. C. Parker, and S. D. Walker, "Polarization Dependent Loss and Temperature Fluctuations Effect on Degree of Orthogonality in Polarization Multiplexed Arrayed Waveguide Grating Based Distribution Networks," IEEE Journal of Quantum Electronics, vol. 41, pp. 945 - 950, 2005. 